\title{
Behaviour of curved reinforcement bars
}

\author{
Mikkel Frydenlev Langberg ${ }^{1}$, Jakob Fisker ${ }^{2}$, Lars German Hagsten ${ }^{3}$, Christian Svarre ${ }^{4}$ \\ Department of Engineering, Aarhus University, Aarhus, Denmark \\ E-mail:2jafi@ase.au.dk (corresponding author)
}

\begin{abstract}
Bended or curved reinforcement bars are often met in assessment of existing reinforced concrete structures and are used in design of nodal regions such as frame corners. Although being present in many tested reinforced concrete structures, only little experimental work has been devoted specifically to the behaviour of curved bars and its interaction with the surrounding concrete. To contribute to a better understanding of this behaviour and functioning of curved reinforcement bars, a preliminary experimental programme has been conducted, the results of which are presented in this paper. The tested specimens were all 90-degree V-shaped beams subjected to constant bending, with the frame corner representing the region of interest. The varied parameter was the statical-height of the adjoining beam segments. The application of optical fibres, mounted on the curved reinforcement, allowed for assessment of the interaction between the curved bars and the concrete. This includes strain/stress variations from which the corresponding tension forces are estimated. The bar/concrete-interaction is qualitatively assessed based upon the gradient of the tension force. Photogrammetric measurements allowed for a detailed study of the accompanying crack development, which showed an area with few cracks at the corner region and a change in inclination of cracks near the corner region.
\end{abstract}

Keywords: reinforced concrete, experimental observations, optical fibre, bond, photogrammetry.

\section{Introduction}

Curved reinforcement bars are often met in relation to assessment of existing reinforced concrete structures such as beams and slabs. In such structures curved bars may both be present at intermediate supports of continuous structures where the longitudinal reinforcement is bend-up to accommodate the change in sign of the moment and at end supports (see Figure 1a). The possible beneficial effect from the curved bar on the shear carrying capacity was often neglected due to lack of rational models (Sørensen, 1970). To utilize the beneficial effects in assessment of existing structures, a better understanding of the behaviour of curved bars and its interaction is a necessity.

a)

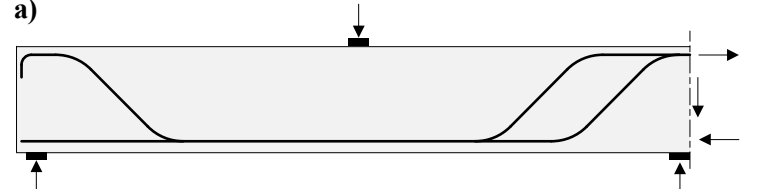

b)

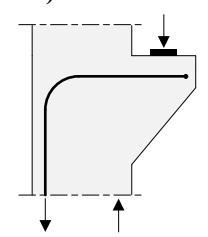

c)

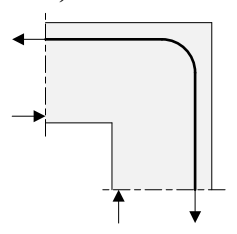

d)

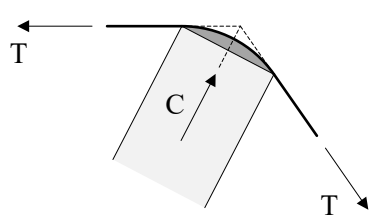

e)

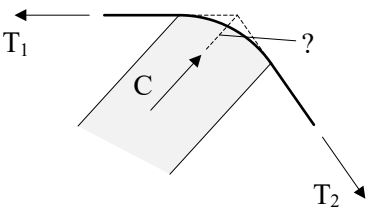

Figure 1. Examples on the use of curved reinforcement bars: a) beam with bend-up longitudinal reinforcement;

b) corbel and c) frame corner. Cases of curved bar nodes: d) symmetric and e) asymmetric

Examples on the use of curved bars today are corbels and frame corners (see Figure 1b-c). Common for all the examples are; the curved bar constitutes the two tension ties of a so-called curved bar node (CTT-node) in which the two tension ties equilibrate a diagonal compressive strut (Klein, 2008). In general, two different cases of curved bar nodes exist for the plane problem which depend on whether the diagonal compressive strut bisects the angle between the two tension ties (the central angle). A symmetric case of a curved bar node is obtained if the diagonal compressive

(C) 2019 Authors. Published by VGTU Press. This is an open-access article distributed under the terms of the Creative Commons Attribution (http://creativecommons.org/licenses/by/4.0/) License, which permits unrestricted use, distribution, and reproduction in any medium, provided the original author and source are credited. 
strut bisects the central angle (see Figure 1d); this is the type of node often dealt with in the theory (see e.g. Muttoni, Schwartz, \& Thürlimann, 1996). In this case the transfer of forces between the curved bar and the surrounding concrete can be attributed to radial stresses of constant magnitude acting normal to the curve of the bar, which produce in combination with the diagonal strut a zone in bi-axial compression between the reinforcement and diagonal strut. On the contrary, only little is known about the distribution of stresses in the asymmetric case illustrated in Figure 1e.

Curved bars have been present in various experimentally tested concrete structures, see e.g. (Swann, 1969; Mayfield, Kong, Bennison, \& Twiston Davies, 1972; Stroband \& Kolpa, 1983; Johansson, 2000) for tests on frame corners and (Özden, 1967) for tests on beams with bend-up reinforcement. Detailed experimental work devoted specifically to the strain/stress distribution along the curved bar has, however, never been conducted.

This paper presents and discusses the results of a preliminary experimental investigation on the behaviour of curved reinforcement bars. The experimental programme consisted of four specimens representing symmetric and asymmetric cases of curved bar nodes. The aim of the tests was to investigate the strain/stress variation along the curved bar and its mechanical behaviour.

\section{Experimental programme}

The experimental programme consisted of four specimens. In the following, reference will be made to each specimen by their number: V-B1 to V-B4. Each specimen was tested in a four-point-bending setup resulting in pure bending at the central part (see Figure 2). The central part of each specimen, which represented an orthogonal frame corner, was analysed during the experiments. The geometry of the specimens is shown in Figure 2 and listed in Table 1. Each specimen was provided with five continuous curved reinforcement bars at the orthogonal corner, with a diameter of $\varnothing=16 \mathrm{~mm}$ and a bend radius of $r_{1}=100 \mathrm{~mm}$. The varied parameter in the experimental programme was the crosssectional height $\left(h_{2}\right)$ of the right adjoining beam segment, resulting in different angles of the diagonal compressive strut in the curved bar node present in the outer corner. V-B2 represented a symmetric case of a curved bar node, whereas V-B1, V-B3 and V-B4 represented different asymmetric cases. The concrete cover ( $c$ ) was $50 \mathrm{~mm}$ for each specimen. Outside the analysed region, the outer geometry and stirrup spacing of the specimens varied slightly.

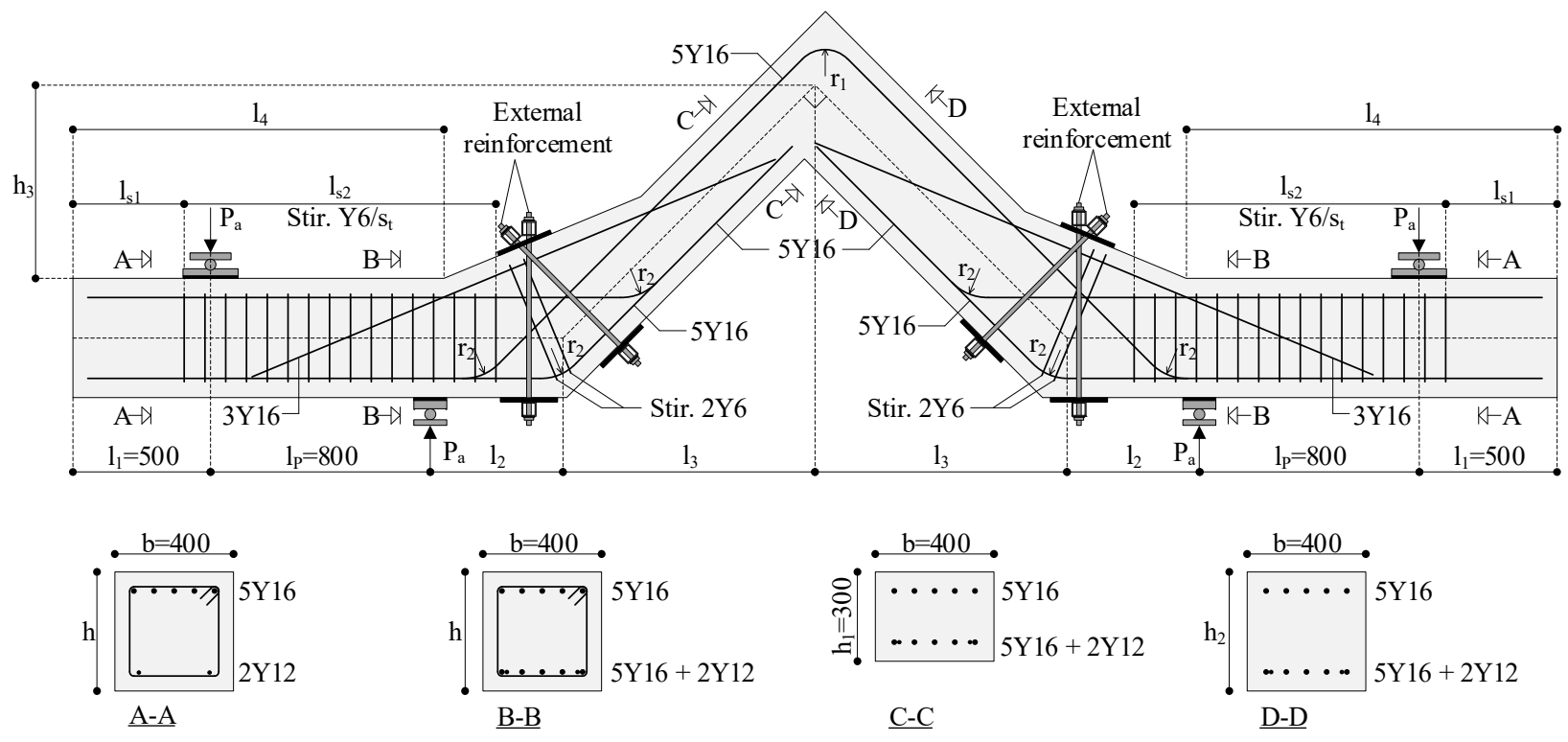

Figure 2. Outer geometry and reinforcement layout of specimens. All dimension are in $\mathrm{mm}$

Table 1. Varying parameters of outer geometry and reinforcement layout of specimens. Measured material properties

\begin{tabular}{|c|c|c|c|c|c|c|c|c|c|c|c|c|c|}
\hline & $\begin{array}{c}h \\
{[\mathrm{~mm}]}\end{array}$ & $\begin{array}{c}h_{2} \\
{[\mathrm{~mm}]}\end{array}$ & $\begin{array}{c}h_{3} \\
{[\mathrm{~mm}]}\end{array}$ & $\begin{array}{c}l_{2} \\
{[\mathrm{~mm}]}\end{array}$ & $\begin{array}{c}l_{3} \\
{[\mathrm{~mm}]}\end{array}$ & $\begin{array}{c}l_{4} \\
{[\mathrm{~mm}]}\end{array}$ & $\begin{array}{c}l_{s 1} \\
{[\mathrm{~mm}]}\end{array}$ & $\begin{array}{c}l_{s 2} \\
{[\mathrm{~mm}]}\end{array}$ & $\begin{array}{c}s_{t} \\
{[\mathrm{~mm}]}\end{array}$ & $\begin{array}{c}E_{s} \\
{[\mathrm{GPa}]}\end{array}$ & $\begin{array}{c}\varepsilon_{y} \\
{[\%]}\end{array}$ & $\begin{array}{c}f_{y} \\
{[\mathrm{MPa}]}\end{array}$ & $\begin{array}{c}f_{c} \\
{[\mathrm{MPa}]}\end{array}$ \\
\hline V-B1 & 300 & 200 & 486 & 564 & 636 & 1450 & 360 & 1080 & 60 & 186 & 0.370 & 577 & 24.7 \\
\hline V-B2 & 300 & 300 & 486 & 564 & 636 & 1450 & 375 & 1060 & 50 & 201 & 0.347 & 569 & 25.4 \\
\hline V-B3 & 400 & 400 & 649 & 351 & 849 & 1250 & 340 & 1120 & 70 & 207 & 0.316 & 537 & 24.9 \\
\hline V-B4 & 500 & 500 & 811 & 139 & 1061 & 1050 & 330 & 1140 & 95 & 186 & 0.351 & 531 & 25.8 \\
\hline
\end{tabular}




\section{Test setup}

Each specimen was simply supported and horizontal movements were permitted at loading points. The distance between the supports was $2400 \mathrm{~mm}$ and the loads were applied with an internal distance of $4000 \mathrm{~mm}(800 \mathrm{~mm}$ of each support). Loading plates were $200 \times 200 \times 25 \mathrm{~mm}$ and support plates were $120 \times 400 \times 30 \mathrm{~mm}$. Neoprene plates with a thickness of $20 \mathrm{~mm}$ was placed between loading/support plates and specimen to avoid stress concentrations.

Additional reinforcement was provided externally at the $135^{\circ}$-corners subjected to opening moments to avoid failure to occur here. The external reinforcement consisted of M16 8.8 threaded rods and SHS $50 \times 3 \mathrm{~mm}$ steel profiles (see Figure 2). Neoprene plates was placed between the SHS profiles and the specimen. The additional reinforcement was not sufficient in order to avoid significant cracking at the $135^{\circ}$-corners for $\mathrm{V}$-B1 and V-B2, however it had no influence on the observed performance of the orthogonal corner.

\section{Instrumentation and measurements}

During the test of each specimen strains were measured along longitudinal reinforcement at the orthogonal corner using fibre optical cables. Fibre optical cables were attached to the top and bottom of reinforcement bars V-Bi-2 and $\mathrm{V}$-Bi-4, see Figure 3. The fibre optical cables had a gauge length of $5 \mathrm{~mm}$ with $50 \%$ overlap, thus strains were measured per 2.5-2.6 $\mathrm{mm}$. The positions along the reinforcement bar and fibre optical cables were calibrated using point $p_{c}$ as reference. Point $p_{c}$ represent the midpoint along the curved part of the reinforcement bar and its position along the fibre optical cables was determined by touching the reinforcement bar with a hot soldering bit at the point of interest before casting the specimens. The hot touch produced localized strains large enough to detect the point in the LUNA software associated with the fibre optical cables. Before any testing commenced it was found that the fibre along the bottom of reinforcement bar V-B1-4 and V-B4-2 had been damaged during casting and was no longer functional. Photogrammetric measurements were performed on the front of the specimen to track cracking. Displacement transducers measured (in and out of plane) displacements as shown in Figure 3.

\section{Materials}

The specimens were cast in one batch together with 12 concrete cylinders (with a diameter of $100 \mathrm{~mm}$ and a height of $200 \mathrm{~mm}$ ). Three cylinders were tested the same day as the testing of the specimens. The mean compressive strengths are listed in Table 1 . The maximum aggregate size of the concrete was $16 \mathrm{~mm}$. Longitudinal reinforcement was hot-rolled quality B550BR and had a well-defined yield plateau. Samples of each of the curved bars were tested to determine their stress-strain relation for further analysis. The modulus of elasticity, yield strain and mean yield strength of the tested bars are summarized in Table 1.

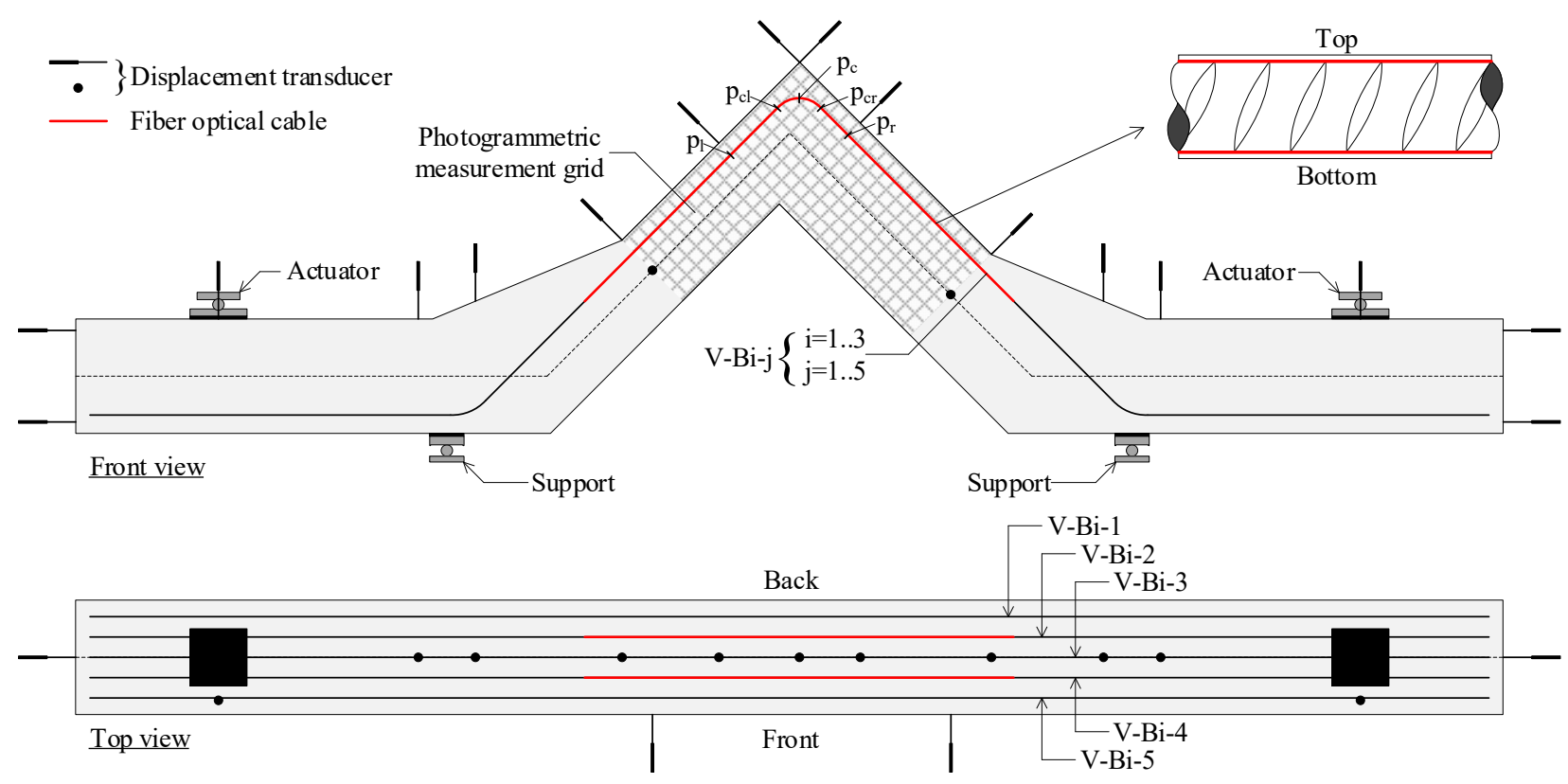

Figure 3. Test setup and instrumentation shown on a front and top view of a specimen. Fibre optical cables were glued to the top and bottom of two of the curved reinforcement bars at the orthogonal corner as indicated by the red lines

\section{Testing and crack development}

Figure $4 \mathrm{a}-\mathrm{d}$ show the force-time diagram of each test. Initially each specimen was subjected to a loading/unloading-cycle with a maximum force of $50 \%$ of the expected flexural capacity of the weakest adjoining beam segment. The 
expected flexural capacity was determined as the theoretical flexural strength (assuming a rectangular stress block in the concrete and yielding of the longitudinal reinforcement) of the adjoining beam segment with the smallest height and is in the following used as a reference capacity of each specimen. Reference capacities $\left(P_{\text {flex }}\right)$ are listed in Table 2.

The load procedure was stopped at certain rates of the reference capacity to conduct strain measurements using the fibre optical cables indicated by the black circles. The black dot indicates the last strain measurement that has been used for further analysis. Strain measurements following this load level showed excessive measurement spikes and were therefore discarded. Measurement spikes are further discussed in the measurement spikes section.

$\mathrm{V}-\mathrm{B} 3$ was tested twice as shown in Figure 4c. The first test was stopped before peak was reached due to an error in the control of the actuators. The configuration of the actuators was corrected before the second test.

In Figure 4e-h the state of cracking is shown for each of the specimens, where the colouring of cracks corresponds to the coloured dots in Figure 4a-d. From the figure, it is seen that almost no cracks occurred within the region bounded by the curved part of the reinforcement bar and the inner corner. Furthermore, the cracks away from the corner region tend to be almost orthogonal to the concrete surface and results must likely from bending, whereas the inclination of the cracks closet to the corner region is significantly different.

The cracks parallel with the beam axis situated at the compressive zone can be observed to occur at and after peak was reached and resulted in gradual spalling of concrete as the deformations increased. For V-B1 and V-B2 excessive cracking can be observed to extend from the $135^{\circ}$-corner towards the orthogonal corner, see Figure $4 \mathrm{e}-\mathrm{f}$.

The moment-rotation diagrams shown in Figure 5a illustrates the relative rotations between two sections $200 \mathrm{~mm}$ away from the corner region plotted against the moment at the central part of the test setup. The relative rotations are based on the photogrammetric measurements. From the figure, it is observed that test 1 on V-B3 was stopped before any significant plastic deformations at the orthogonal corner. Furthermore, it can be seen that the stiffness of V-B1 is significantly lower than the others, which is caused by the smaller height of the weakest adjoining beam segment.
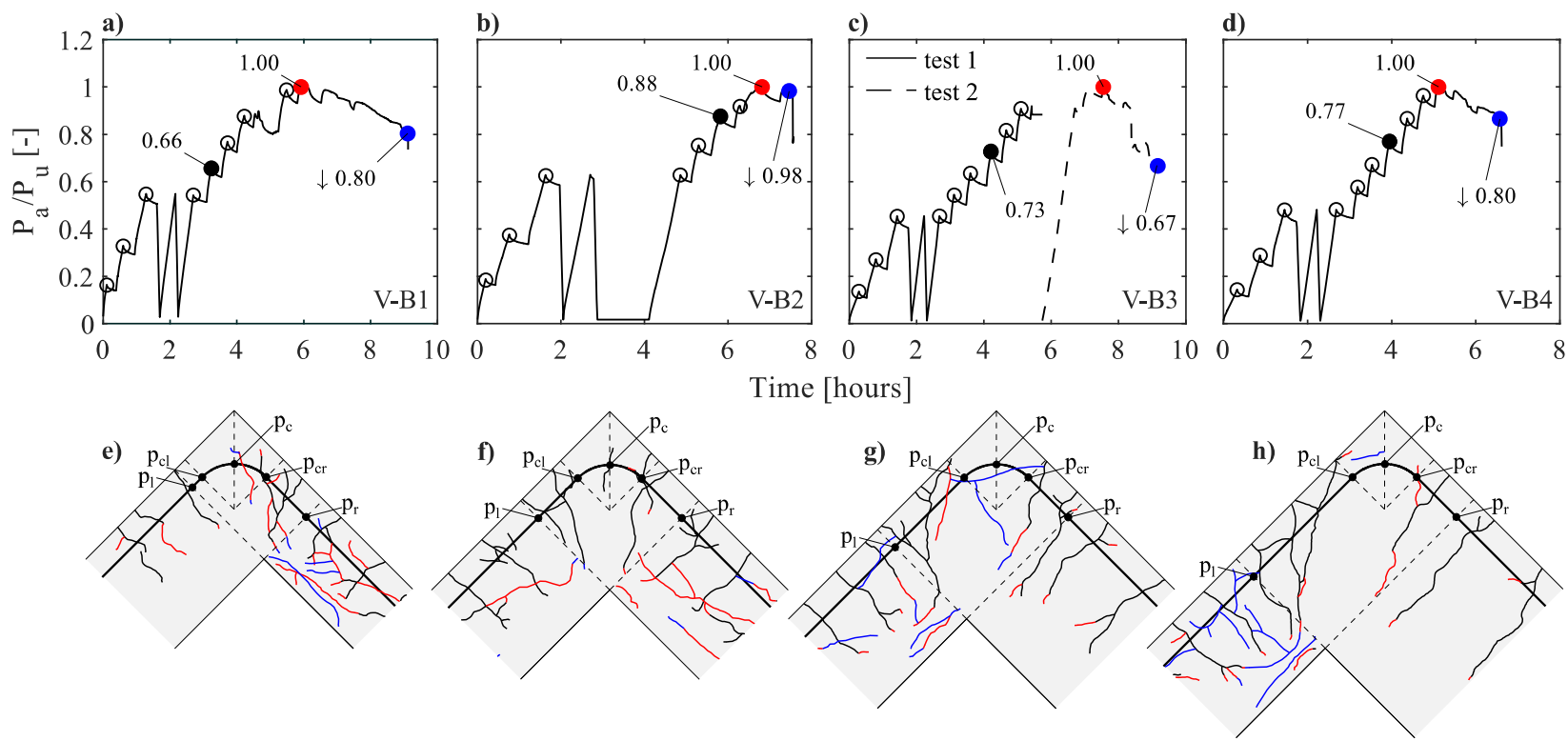

Figure 4. a)-d) Force-time diagrams for each specimen. Black circles indicates strain measurements.

Black dot indicates last strain measurement that has been used for further analysis.

Red dot indicates peak load. Blue dot indicates load level where unloading commenced;

e)-h) State of cracking at the orthogonal corner. Colouring of cracks corresponds to the coloured dots in Figure 4a)-d)

Table 2. Reference capacity, experimental peak load and failure mode of each specimen

\begin{tabular}{|c|c|c|c|l|}
\hline & $P_{\text {flex }}$ & $P_{u}$ & $P_{u} / P_{\text {flex }}$ & \multirow{2}{*}{ Failure mode } \\
\cline { 2 - 4 } & {$[\mathrm{kN}]$} & {$[\mathrm{kN}]$} & {$[-]$} & \\
\hline V-B1 & 80.1 & 67.7 & 0.85 & Flexural (at 135 -corner) \\
\hline V-B2 & 151.3 & $(114.1)$ & $(0.75)$ & Terminated before failure \\
\hline V-B3 & 143.6 & 157.8 & 1.10 & Flexural \\
\hline V-B4 & 142.9 & 148.9 & 1.04 & Flexural \\
\hline
\end{tabular}




\section{Failure mode}

In Table 2 the applied load at failure $\left(P_{u}\right)$ is listed together with the ratio between the reference capacity and load at failure $\left(P_{u} / P_{f l e x}\right)$. Specimens V-B3 and V-B4 failed in a flexural manner with concrete crushing at the compressive zone of the weakest adjoining beam segment. The concrete crushing was located near the orthogonal corner (see Figure $4 \mathrm{~g}-\mathrm{h}$ ). This failure mode resulted in ratios around $P_{u} / P_{\text {flex }}=1$. On the other hand, the peak load of V-B1 and $\mathrm{V}-\mathrm{B} 2$ can be seen from Table 2 to be less than the reference capacity because failure occurred at the $135^{\circ}$-corners for V-B1 while V-B2 was terminated before failure occurred.

\section{Discussion of test results}

In the following, the test results are discussed with focus on the strain measurements from the fibre optical cables and the state of cracking based on the photogrammetric measurements. Position of strain measurements along the fibre optical cables are based on the nominal geometry.

\section{Measurement spikes}

Figure $5 \mathrm{~b}$ show the strains measured along the top of reinforcement bar V-B4-4. Measurement spikes can be observed where cracks intersect the reinforcement bar (compare with Figure $4 \mathrm{~h}$ ) and at the ends of the curved part (near points $p_{c l}$ and $p_{c r}$ ). These observation could be made for all strain measurements to both a lesser and greater extend. Measurement spikes represent values or variations of strains outside the range of strains that should be expected. Any measured strains above 2 times the yield strain have been removed. In addition, strains resulting in extreme variations have been removed. Extreme variations have been regarded as the difference in two adjacent measurements greater than $20 \%$ of the yield strain. In Figure $5 \mathrm{c}$, the same measured strains are shown with the measurement spikes removed. For further analysis, linear interpolation was made over the missing measurement ranges. For some fibre optical cables and some load levels the strain measurements were discarded because the distribution of measurement spikes was too excessive.
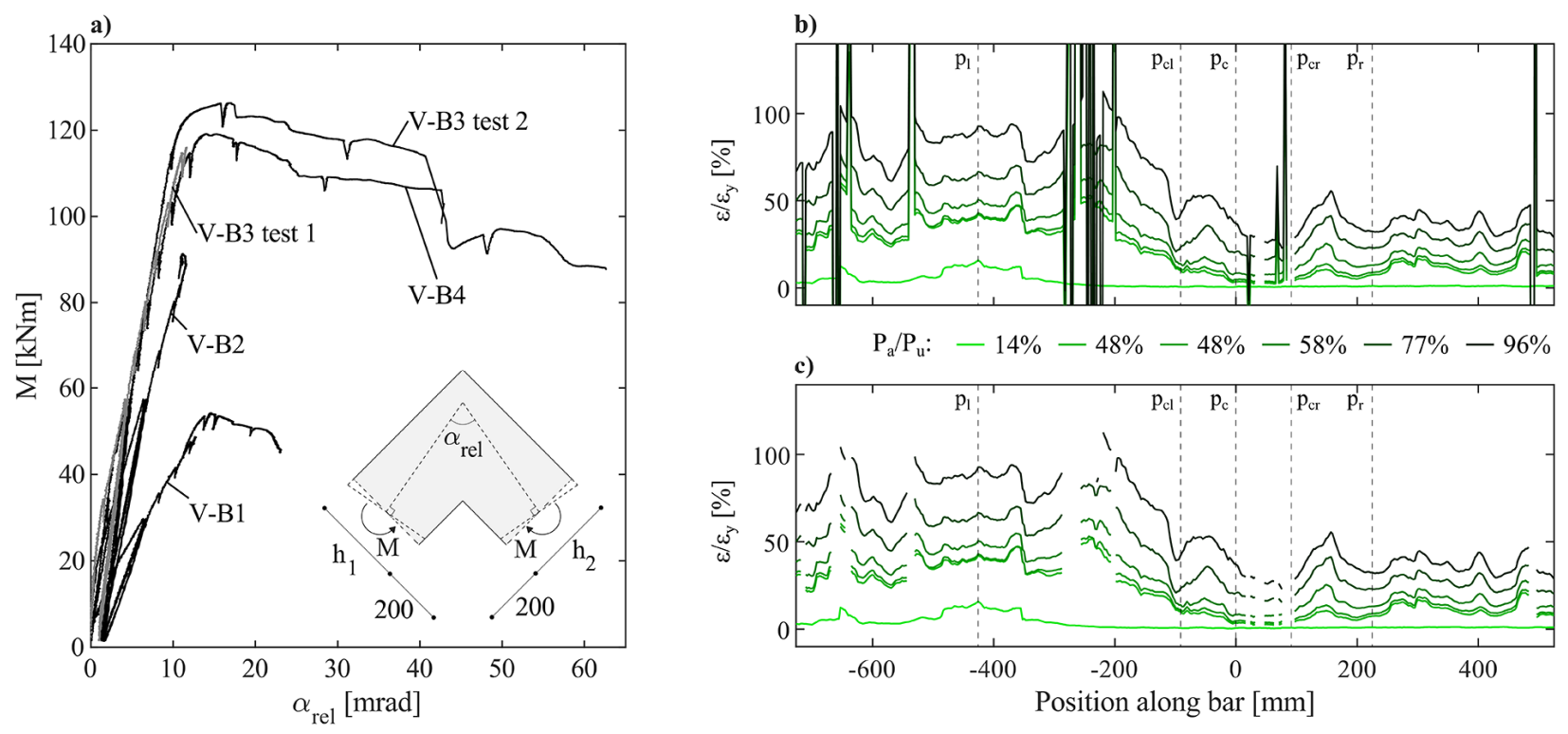

Figure 5. a) Moment-rotation diagrams for each specimen. Strain measurements along the top of bar V-B4-4; b) w/ and c) w/o measurement spikes

\section{Mean strains}

The strains measured along the two bars in each specimen was generally similar, why the strains shown in the following are the mean of the two bars. In Figure 6a-d the mean of the measured strains are shown. For the load-level indicated by the black dot in Figure 4a-d the mean of the strains measured by all four fibres are shown by the black line, while the green and blue line indicates the mean of strains measured by the two fibres along the top and bottom, respectively. The grey lines show the mean strains of all four fibres for preceding load-levels.

For all four specimens, it is seen that the strains at the top and bottom have rather large variations, even along the straight parts. However, they tend to have opposite extrema at the same position, which result in approximately constant strain-levels along the straight parts of the bar when the mean of all four fibres is considered. For the symmetric case 
(V-B2) the strain-levels on each side of the curved part is almost equal, whereas they are significantly different for the asymmetric case (V-B1, V-B3 and V-B4), where the greater strain-level is located at the side of the weakest adjoining beam segment. For V-B2 an abrupt decrease in strains can be observed at the ends of the curved part and an almost constant strain-level within the curved part. A distinct variation of the strains can be observed for the remaining specimens, where the strains gradually decrease to a local minimum within the curved region and increase to the strainlevel on the opposite side. The decrease in strains is restricted to the curved part for all specimens apart from V-B4, where the decrease in strains extend significantly beyond the curved part.
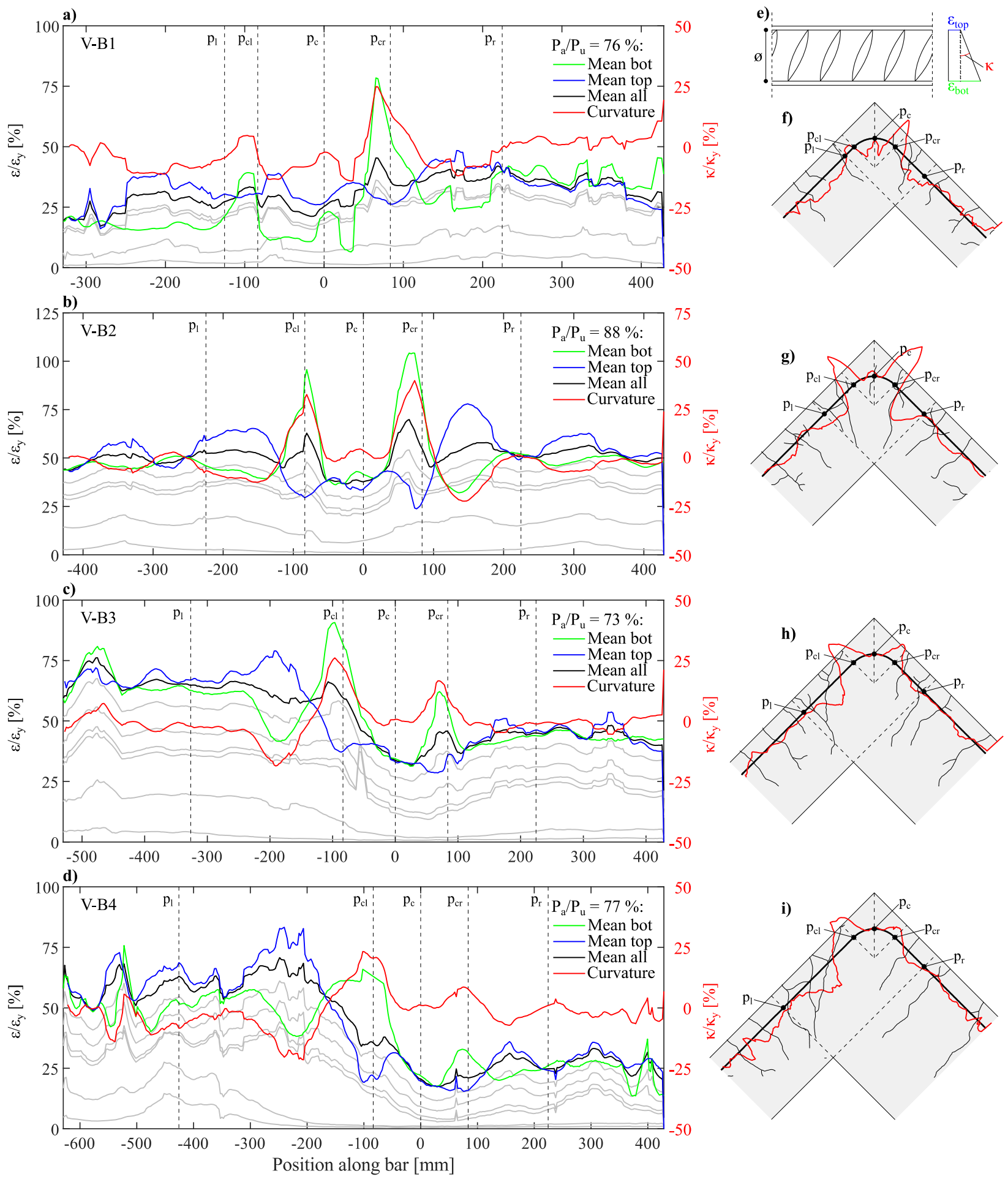

Figure 6. a)-d) Variation of measured strains and estimate on curvature; e) Positive definition of curvature; f)-i) Curvature plotted normal to reinforcement bar. State of cracking corresponds to black dot in Figure 4a-d 
The variation of mean strain (black and grey line) is very similar for different load-levels and only small strain increments can be observed after the loading/unloading-cycle, which agrees with the fact that almost no strains above the yield strain is observed. Thus, the local minimum of strains within the curved part of the bar tend to occur at the same location for all load-levels. Furthermore, the local minimum tend to be located closer to the strongest of the adjoining beam segments. The same observation as mentioned above could be made for the variation of stresses as well, because yielding of the bars had not occurred, why the stresses would simply be a scaling of the strains.

\section{Curvature}

Differences around $50 \%$ of the yield strain can be observed between the measured strains at the top and bottom of a reinforcement bar. These large variations might be caused by bending experienced by the bar itself. Using the mean of strains measured by the two fibres along the top and bottom, an estimate of the curvature of the bar has been determined. The curvature is positively defined as shown in Figure 6e. In Figure 6a-d the variation of the relative curvature is shown $\left(\kappa_{y}\right.$ is the curvature that results in yielding in tension and compression at the bottom and top fibre of the bar, respectively). In Figure $6 \mathrm{f}-\mathrm{i}$ the curvature is plotted normal to the bar, to illustrate where large curvatures occur. From the figure, it is noted that large positive curvatures occur at the ends of the curved part (points $p_{c l}$ and $\left.p_{c r}\right)$. Furthermore, large negative curvatures occur between points $p_{l}-p_{c l}$ and $p_{c r}-p_{r}$.

\section{Tension force}

Figure $7 \mathrm{a}-\mathrm{d}$ shows an estimate on the tension force carried by all five reinforcement bars for each specimen. The tension force is based on the mean of the measured strain for all four fibres and the stress-strain relation determined from the material tests. It is noted that the variation of the tension force is simply a scaling of the variation of the strains, because yielding did not occur. Thus, the tendencies observed for the measured strains apply for the tension force as well.
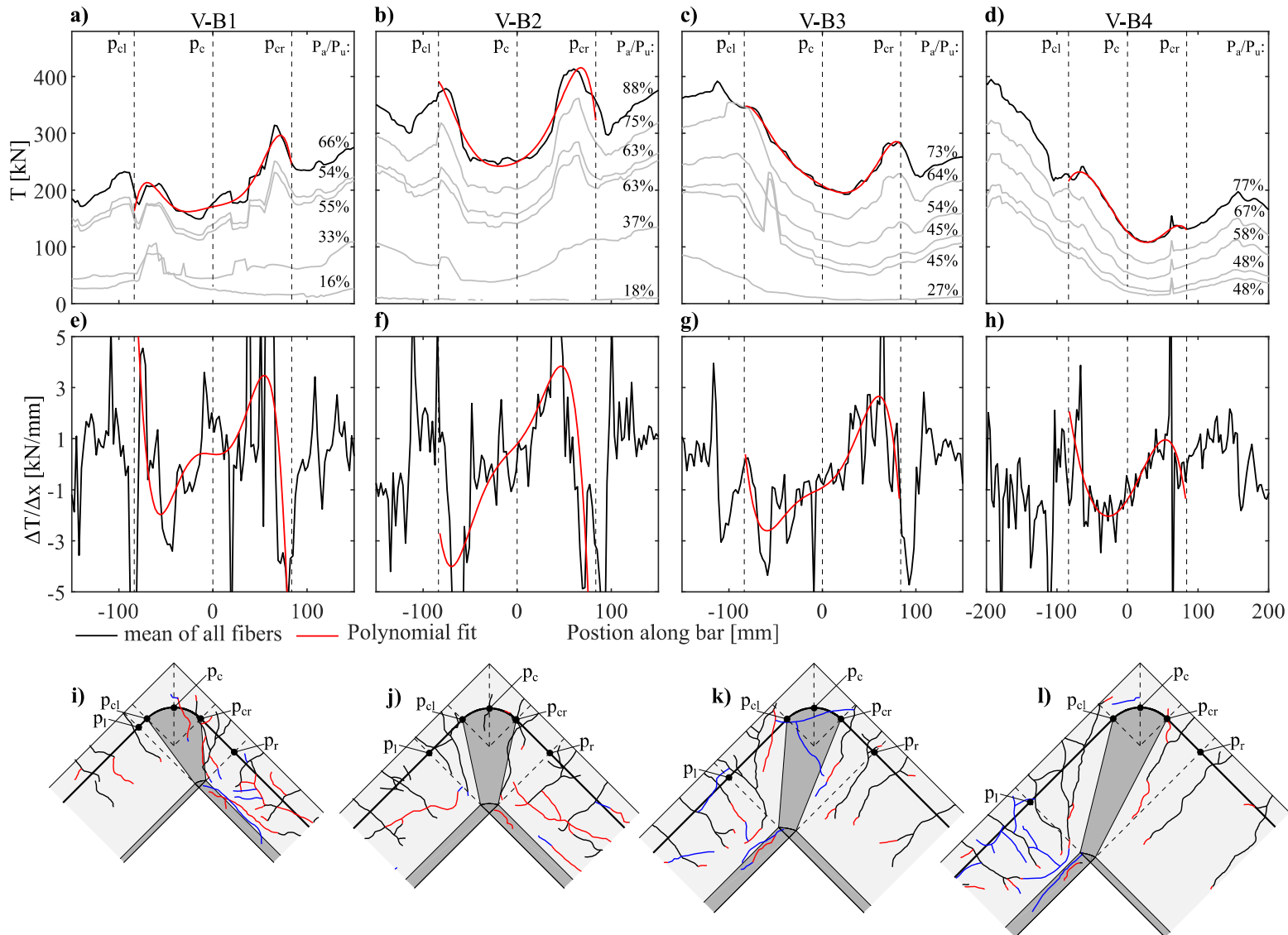

Figure 7. Variation of a)-d) tension force and e)-h) gradient of the tension force in the curved part of the reinforcement bar; i)-1) State of cracking and a possible stress field at the orthogonal corner. Colouring of cracks corresponds to the coloured dots in Figure 4a-d

\section{Concrete interaction}

The variation of strains within the curved part of the bar and thus the tension force is a result of the combination of the bar/concrete-interaction and bending experienced by the bar itself. The bar/concrete-interaction are the result of 
bond and radial stresses at the interface and estimates on these are associated with model assumptions. Qualitatively the gradient of the tension force is an estimate on the bar/concrete-interaction where no model assumptions are needed for its determination. The gradient of the tension force has been determined as the difference in two adjacent tension forces divided by the distance between them. This results in the rather scattered gradient of the tension force shown in Figure 7e-h (black line). $6^{\text {th }}$ order polynomials were fitted to the variation of the tension force in the curved part (see Figure 7a-b) to obtain an improved estimate on the variation behind the scattered data (see Figure 7e-h). From the figure, it is seen that the absolute magnitude of the gradient is largest at the ends of the curved part (at points $p_{c l}$ and $p_{c r}$ ) which indicates a large interaction, while the smallest interaction is at the point of the local minimum of strains, which is also at the point where the gradient changes sign.

In Figure 7i-1 the state of cracking is shown together with a possible diagonal compressive strut or stress field. It can be observed that the sides of the strut have similar inclinations as the cracks closet to the corner region. Assuming that the strut only carries uniaxial compressive stresses along radial stress trajectories in a fan-shaped manner, a stress trajectory will be orthogonal to the curved bar at a point located closer to the strongest of the adjoining beam segment, which seem to be near the point of local minimum of strains. The stress field predicts no bond stresses at this point, and the bar/concrete-interaction would be minimum, which in fact agrees with the observation made from Figure 7eh. Furthermore, the difference between the inclination of the normal to the reinforcement bar and the stress trajectories are greatest at the ends of the curved part, why large bond stresses are predicted by the stress field here, which results in large bar/concrete-interaction, likewise in agreement with Figure 7e-h.

\section{Conclusions}

This paper summaries the results of a preliminary experimental investigation on the distribution of strains/stresses in curved reinforcement bars and its mechanical behaviour. The main conclusions are:

- Scattered but reliable strain measurements can be obtained using fibre optical cables.

- Bar strains vary within the curved part with decreasing magnitude from each side and attain a local minimum that tends to be located closer to the strongest of the adjoining beam segments.

- Differences of up to $50 \%$ of the yield strain were observed between the top and bottom of a bar, which may indicate significant bending of the reinforcement bar itself.

- Estimates on the curvature of the bar shows large positive curvatures within the curved part and large negative curvatures along the straight parts within the corner region.

- A region with only few cracks could be observed at the orthogonal corner. The state of cracking indicated a possible applicable compressive strut within the corner region bounded by the intersecting compressive zones of each adjoining beam segment and the curved bar.

- The gradient of the tension force indicates large bar/concrete-interaction at the ends of the curved part of the bar.

\section{Acknowledgements}

The experimental work presented in this paper was conducted by Jensen, E. and Susgaard, M. as a part of their Master's Thesis "Investigation of Stresses and Behavior of Curved Bars in Reinforced Concrete Frame Corners", (see Jensen \& Susgaard, 2019).

\section{References}

Jensen, E., \& Susgaard, M. (2019). Investigation of stresses and behavior of curved bars in reinforced concrete frame corners (Master's thesis). Aarhus University.

Johansson, M. (2000). Structural behaviour in concrete frame corners of civil defence shelters (PhD dissertation). Chalmers University of Technology.

Klein, G. J. (2008). Curved-bar nodes. A detailing tool for strut-and-tie models. Concrete International. $17 \mathrm{p}$.

Mayfield, B., Kong, F.-K., Bennison, A., \& Twiston Davies, J. C. D. (1971). Corner joint details in structural lightweight concrete. International Concrete Abstracts Portal, ACI, 68(5), 366-372. https://doi.org/10.14359/11336

Muttoni, A., Schwartz, J., \& Thürlimann, B. (1996). Design of concrete structures with stress fields. Berlin: Birkhäuser. https://doi.org/10.1007/978-3-0348-9047-2

Özden, K. (1967). An experimental investigation on the shear strength of reinforced concrete beams. Technical University of Istanbul.

Sørensen, H. C. (1970). Forskydning i jernbetonbjcelker (Report R 19). Technical University of Denmark, Copenhagen.

Stroband, J., \& Kolpa, J. J. (1983). The behaviour of reinforced concrete column-to-beam joints, Part 1: Corner joints subjected to negative moments (Report 5-83-9, Stevin Laboratory). Delft University of technology.

Swann, R. A. (1969). Flexural strength of corners of reinforced concrete portal frames (Technical report TRA 434). Cement and Concrete Association, London. 